\title{
Clindamycin Resistance among Methicillin Resistant Staphylococcus aureus Isolated from Human and Respective Household Swine in Greater Kabale Region-South Western Uganda
}

\author{
Andrew Baguma ${ }^{1}$, Benson Musinguzi1,2, Moses Mpeirwe1 ${ }^{1}$, Joel Bazira1 \\ ${ }^{1}$ Department of Microbiology, Faculty of Medicine, Mbarara University of Science and Technology, \\ Mbarara, Uganda \\ ${ }^{2}$ Department of Medical Laboratory Sciences, School of Allied Health Sciences, Kampala International University, \\ Bushenyi, Uganda \\ Email: `2014phd004@std.must.ac.ug
}

How to cite this paper: Baguma, A., Musinguzi, B., Mpeirwe, M. and Bazira, J. (2019) Clindamycin Resistance among Methicillin Resistant Staphylococcus aureus Isolated from Human and Respective Household Swine in Greater Kabale RegionSouth Western Uganda. Advances in Infectious Diseases, 9, 285-294.

https://doi.org/10.4236/aid.2019.94022

Received: November 1, 2019

Accepted: December 9, 2019

Published: December 12, 2019

Copyright $\odot 2019$ by author(s) and Scientific Research Publishing Inc. This work is licensed under the Creative Commons Attribution International License (CC BY 4.0).

http://creativecommons.org/licenses/by/4.0/

(c) (i) Open Access

\begin{abstract}
Introduction: $S$. aureus is recognized as the common cause of nosocomial and community-acquired infections. Macrolide-Lincosamide-Streptogramin $\mathrm{B}\left(\mathrm{MLS}_{\mathrm{B}}\right)$ is thought to be alternative therapies against MRSA infections. Clindamycin is the most favored agent because of exceptional pharmacokinetic characteristics. However, increasing resistance to clindamycin among MRSA strains is a serious challenge. The current study investigated the profile of clindamycin resistance among MRSA isolates from Humans, and their respective livestock (in particular swine) using D-test in greater Kabale region. Materials and Methods: Three hundred phenotypic MRSA isolates previously isolated from Humans and swine were confirmed by mecA PCR. We performed D-test using erythromycin $(15 \mu \mathrm{g})$ and clindamycin $(2 \mu \mathrm{g})$ discs in accordance to Clinical and Laboratory Standards Institute (CLSI) protocol. Results: Of all 300 MRSA isolates, $6 \%(n=18)$ were sensitive to Erythromycin and Clindamycin (S). The rate of inducible clindamycin resistance (iMLSB) was $42 \%(\mathrm{n}=125)$ and $38 \%(\mathrm{n}=115)$ was resistance to both Erythromycin and clindamycin (cMLSB). However, $14 \%(n=42)$ were resistant to erythromycin but sensitive to clindamycin (MS) without " $D$ " zone negative. Conclusion: Clindamycin resistance (both cMLSB and iMLSB) among MRSA was high and " $D$ " test should be adopted routinely during antimicrobial susceptibility testing by disc diffusion testing to rapidly detect iMLSB and CMLSB.
\end{abstract}




\section{Keywords}

Clindamycin Resistance, cMLSB and iMLSB Phenotypes, MRSA

\section{Introduction}

S. aureus is recognized as one of the most common organisms causing nosocomial and community-acquired infections worldwide. The emergence of multidrug resistant $S$. aureus strains, especially methicillin resistant $S$. aureus (MRSA), is of a particular concern. In Uganda, this has been largely attributed by empirical antibiotic prescriptions [1]. The cumulative MRSA problem is an indicator for urgent need for new antibiotics.

Macrolide-Lincosamide-Streptogramin B $\left(\mathrm{MLS}_{\mathrm{B}}\right)$ antibiotics have been thought about as alternative solution to treat MRSA infections [2]. The most commonly used antibiotic in the MLSB group are the macrolides (e.g. erythromycin and azithromycin) and clindamycin which is a Lincosamide [3].

Macrolides act through inhibition of protein synthesis by binding irreversibly to the 23S ribosomal RNA (rRNA) on the bacterial 50S ribosomal subunit and subsequent disruption of the growing peptide chain by blocking translocation [4]. Lincosamide [e.g. clindamycin and lincomycin] bind to the 50S ribosomal subunit and prevent peptide elongation by interfering with the peptidyl transfer during protein synthesis [5]. Clindamycin is the most preferred agent because of exceptional pharmacokinetic characteristics [6] and is regularly used in the management of severe infections, caused by macrolide resistant $S$. aureus infections including MRSA [7]. However, increasing resistance to clindamycin among MRSA strains and other Staphylococcus is a serious challenge [8]. The expression of clindamycin resistance in Staphylococcus species can be constitutive or inducible [9] [10] through erm genes which codes for ribosomal methylases [11]. In addition, the resistance to the lincosamides (clindamycin), macrolides (erythromycin), and streptogramins (quinupristin/dalfopristin) is facilitated by three related genes, ermA, ermB, and erm C, that encode for erythromycin resistance methylases [12]. Methylase enzymes binds on to the ribosome resulting in a conformational change or modification in the ribosomal target and consequently, decreasing the ability of these drugs to bind to the ribosome [5] [13].

Inducible Clindamycin resistance (iMLSB) cannot be identified by standard methods of antibiotic susceptibility testing and failure to detection may result into treatment failure with Clindamycin [6]. Erythromycin-resistant staphylococci are routinely considered to be resistant to Clindamycin by clinicians [14], a phenomenon that is wrong that shuns Clindamycin prescription to patients infected with macrolide-resistant isolates that may be sensitive to Clindamycin. It is rational to routinely test for presence of iMLSB strains and this can be achieved cheaply by use of Erythromycin and clindamycin discs placed adjacent 
to each other during routine antibiotic sensitivity testing by Kirby Bauer technique for $S$. aureus [2] [15] [16]. Data on profiles of clindamycin resistance among MRSA in Kabale region-South western Uganda is not available. Therefore, the current study investigated the profile of clindamycin resistance among MRSA isolates from Humans, and their respective livestock (in particular swine) using D-test.

\section{Materials and Methods}

This was cross-sectional study, where 300 phenotypic MRSA isolates previously isolated from humans $(\mathrm{n}=200)$ and swine $(\mathrm{n}=100)$ during the period of January 2015 to June 2016 and stored in glycerol $(20 \% \mathrm{v} / \mathrm{v})$ at $-80^{\circ} \mathrm{C}$.

\subsection{DNA Extraction}

These isolates, were subjected to DNA extraction following Queipo et al. and Teeraputon et al., techniques [8] [17] by boiling using $100 \mu \mathrm{l}$ of the bacterial suspension in $1.5 \mathrm{ml}$ cryogenic vials (Eppendorf, Germany) followed by centrifugation at $3000 \mathrm{rpm}$ for 15 minutes. The supernatant was removed, and the pellet suspended using molecular biology-grade water (Eppendorf, Germany) and re centrifuged at $3000 \mathrm{rpm}$ for $10 \mathrm{~min}$. The supernatant was discarded, and the pellet suspended in $100 \mu \mathrm{l}$ of molecular biology-grade water. The suspension was subjected to boiling at $100^{\circ} \mathrm{C}$ for $10 \mathrm{~min}$, cooled on ice, and centrifuged at 15,000 $\mathrm{rpm}$ for 10 seconds before it was stored at $-20^{\circ} \mathrm{C}$.

\subsection{PCR Amplification}

Aliquots of $2 \mu \mathrm{l}$ of template DNA was used for PCR to detect and amplify mecA gene with origonucleotide primer mecA F;

5-TCCAATTACAACTTCACCAGG-3 and mecA R

5-CCACTTCATATCTTGTAACG-3 synthesized by GenxBio to confirm their MRSA status. The reaction mixture $(25 \mu \mathrm{l})$ consisting of $100 \mathrm{pmol}$ of each primer, Taq polymerase (2.5 U), $\mathrm{Mg}^{2+}(2.5 \mathrm{mM}), 2.5 \mu \mathrm{l}$ PCR buffer and $3 \mu \mathrm{ltem}-$ plate DNA. The PCR program was as follows: $3 \mathrm{~min}$ at $94^{\circ} \mathrm{C}$; followed by 40 cycles of a 30 seconds denaturation step at $94^{\circ} \mathrm{C}$, a 30 second annealing step at $45^{\circ} \mathrm{C}$ and a 30 second extension at $72^{\circ} \mathrm{C}$; and a final 10 minutes extension step at $72^{\circ} \mathrm{C}$. The amplified product was a 533 bp sequence, which was detected by $1 \%$ agarose gel electrophoresis with $(0.5 \mathrm{mg} / \mathrm{L})$ ethidium bromide stain and observation under UV light. All strains positive for the mecA gene and designated MRSA. MecA positive (ATCC 43300) and MecA negative (ATCC 29213) were used as positive and negative controls.

\subsection{Detection of Phenotypic Clindamycin Resistance}

To detect clindamycin resistance, a suspension of 0.5 McFarland standard equivalent was prepared from all genotypically confirmed MRSA isolates and a lawn culture of bacterial suspension was seeded on to sterile Muller Hinton Agar 
(MHA) plates. Onto the seeded plates, disk of Clindamycin $(2 \mu \mathrm{g})$ and Erythromycin $(15 \mu \mathrm{g})$ was place in approximately $15 \mathrm{~mm}$ apart (measured edge to edge). The inoculated plates were further incubated at $37^{\circ} \mathrm{C}$ for 16 to 18 hours. The zone of clearance with flattening characteristics (D-shaped) around clindamycin in the area between the two adjacent discs, indicated iMLSB [16].

\subsection{Media Quality Control}

Before use, Mueller Hinton agar (MHA) was quality controlled (QC) by checking its physical appearance, sterility after preparation and capacity to support growth. Sterility testing was performed on $5 \%$ of each batch of new medium prepared in house media selected randomly and incubated for 48 hours at $35^{\circ} \mathrm{C}$ to $37^{\circ} \mathrm{C}$ under aerobic conditions to check for contamination. Fertility test was performed using QC control strains (Staphylococcus aureus ATCC 25923, Escherichia coli ATCC 25922, Pseudomonas aeruginosa ATCC 27853) and subjected to Kirby-Bauer Susceptibility Testing protocol [2] using three MHA plates from each batch prepared and incubated for $18-24$ hours at $35^{\circ} \mathrm{C}$ to $37^{\circ} \mathrm{C}$ under aerobic atmosphere. All the media used passed quality control measures.

\section{Results}

Among the MRSA isolates $(n=300), 6 \%(n=18)$ of the strains were sensitive to both erythromycin and clindamycin, designated as $\mathrm{S}$ phenotype. However, 38\% $(\mathrm{n}=115)$ MRSA strains showed resistance to both erythromycin (zone size $\leq 13$ $\mathrm{mm}$ ) and clindamycin (zone size $\leq 14 \mathrm{~mm}$ ) and these strains were constitutively resistant to clindamycin and designated as "cMLSB" phenotype. In addition, $42 \%(\mathrm{n}=125)$ showed resistance to erythromycin (zone size $\leq 13 \mathrm{~mm})$ and sensitive to clindamycin (zone size $\geq 21 \mathrm{~mm}$ ) with a "D-shaped" zone of inhibition around. These were identified as inducible clindamycin resistant strains and they were designated as iMLSB. Of note, $14 \%(n=42)$ showed resistance to erythromycin (zone size $\leq 13 \mathrm{~mm}$ ) and sensitive to clindamycin (zone size $\geq 21 \mathrm{~mm}$ ) without "D" zone of inhibition around clindamycin and were designated as $\boldsymbol{M S}$ phenotypes as shown in Figure 1.

Among the MRSA with iMLSB phenotypes, $46 \%(n=92)$ isolates were from humans, 33\% $(\mathrm{n}=33)$ from swine. In addition, cMLSB phenotype, 34\% $(\mathrm{n}=68)$ originated from human while $47 \%(n=47)$ were from swine. However, among the MS phenotypes, $15 \%(n=30)$ and $12 \%(n=12)$ were from human and swine respectively whereas among the $S$ phenotype, $5 \%(n=10)$ were from human and $8 \%(n=8)$ were from swine (Figure 2). D-test was used to rule out cMLSB and iMLSB (D zone present) as shown in Figure 3.

Results of mecA gene PCR where an Amplicon of approximately 180 bp was expected using positive and negative controls are shown in Figure 4.

\section{Discussion}

The increasing frequency of the Staphylococcal infections and their respective 


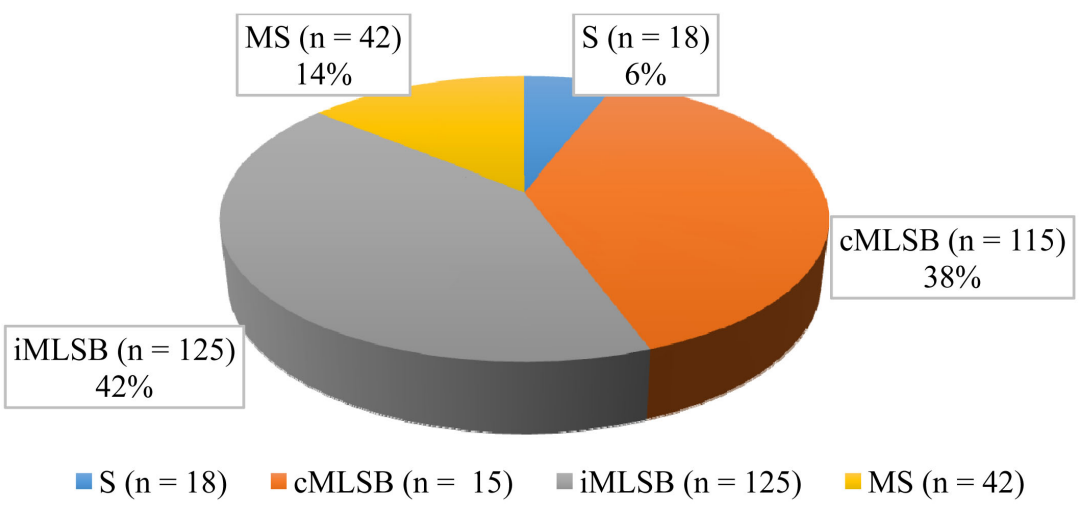

Figure 1. Clindamycin and Erythromycin resistance pattern of MRSA isolates. Footnote: $\mathrm{S}=$ Sensitive to both clindamycin and erythromycin, $\mathrm{cMLSB}=$ constitutive clindamycin; iMLSB = inducible clindamycin resistance (D zone present); MS = Erythromycin resistant but clindamycin sensitive ( $\mathrm{D}$ zone absent).

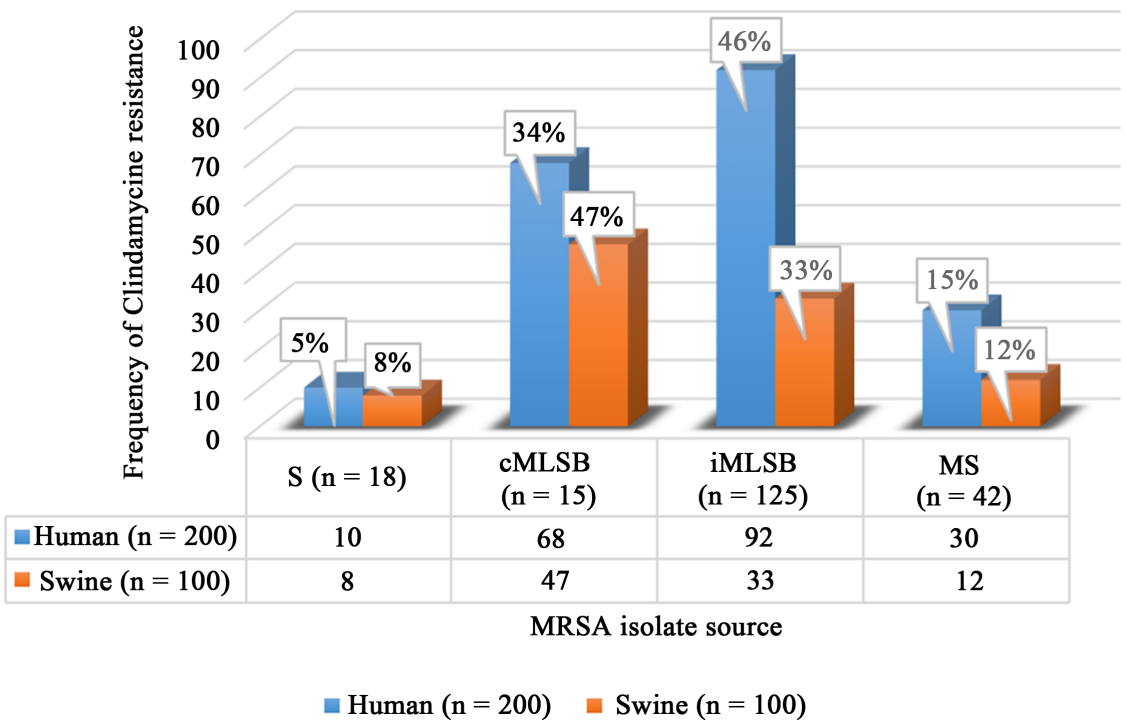

Figure 2. MLSB resistance profile among MRSA isolates. Footnote: $\mathrm{cMLSB}=$ constitutive clindamycin; iMLSB = inducible clindamycin resistance (D zone present); MS= Erythromycin resistant but clindamycin sensitive ( $\mathrm{D}$ zone absent).

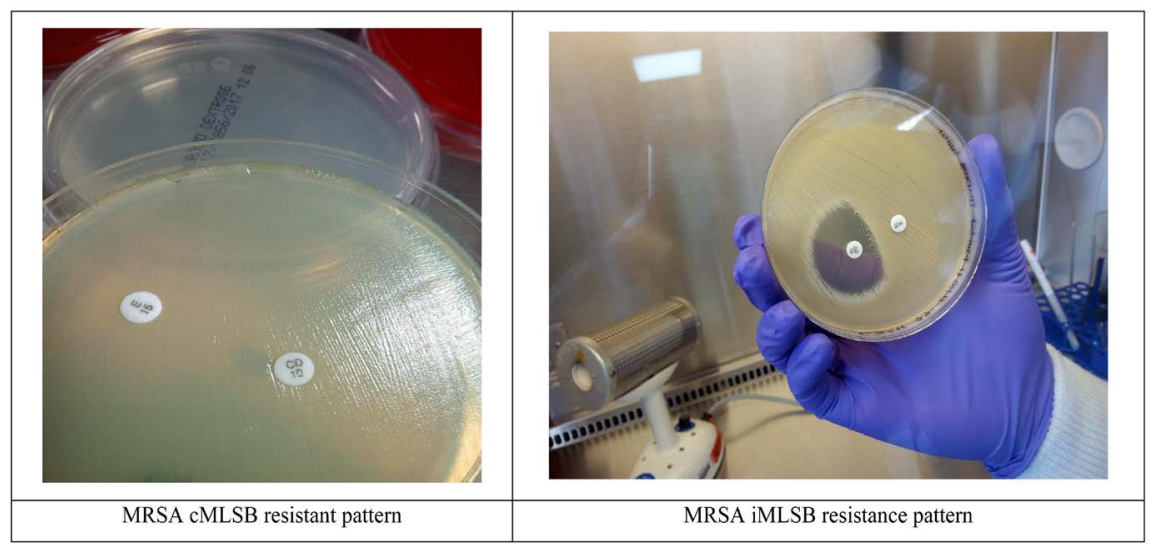

Figure 3. D-test showing inducible clindamycin resistance. 


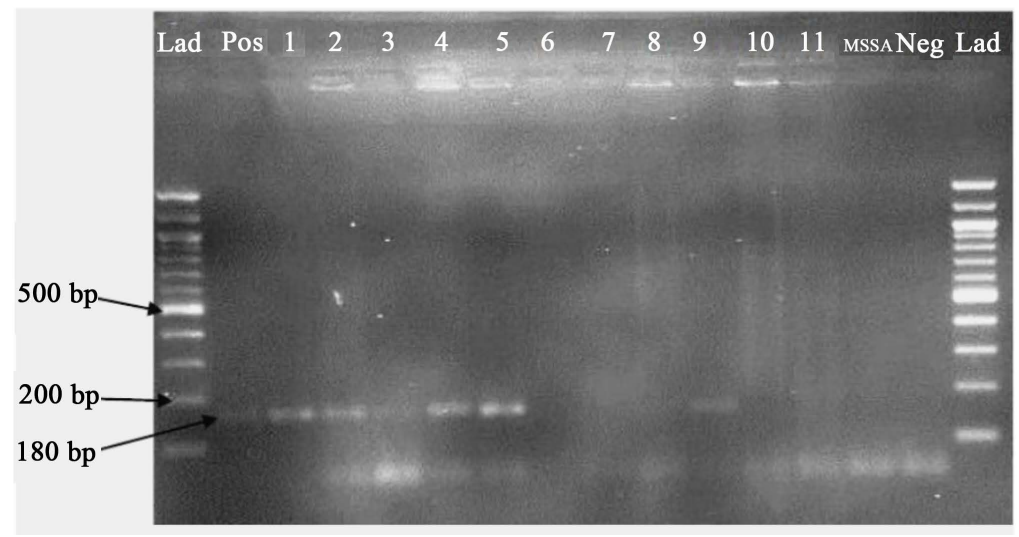

Figure 4. Results of mecA gene PCR. where an Amplicon of approximately 180bp was expected. Lad is $100 \mathrm{bp}$ ladder, Lane Pos. contains the Positive control (MRSA ATCC 43300), and Lanes 1, 2, 3 MRSA isolated from Human 4, 5, and 9 MRSA isolated from swine. Lane 6, 7, 8 MSSA from Human and 10 and 11 were MSSA isolates from swine. MSSA contains a Methicillin Susceptible $S$. aureus strain ATCC 25923, whereas Neg. is the Negative amplification control.

changes in antimicrobial resistance patterns has led to a renewed interest in clindamycin as therapeutic choice. In fact, MRSA prevalence is now regarded as a pandemic scourge with varying prevalence across different countries and among the hospitals as well as livestock population [18] [19]. Indiscriminate use of antibiotics in both human and animal coupled with poor clinical practices may be contributing factors leading to the emergence of MRSA and other antimicrobial resistances(AMRs). Currently, Macrolide-Lincosamide-Streptogramin $\mathrm{B}\left(\mathrm{MLS}_{\mathrm{B}}\right)$ resistance is on the rise and catching public health interest [12]. The Macrolide-Lincosamide-Streptogramin $\mathrm{B}\left(\mathrm{MLS}_{\mathrm{B}}\right)$ resistance may be of the constitutive $\left(\mathrm{CMLS}_{\mathrm{B}}\right)$ or the inducible $\left(\mathrm{iMLS}_{\mathrm{B}}\right)$ type. The isolates with $\mathrm{CMLS}_{\mathrm{B}}$ are resistant to both erythromycin (ER) and clindamycin (CL) and they are readily detected by in vitro testing. This family of antibiotics $\left(\mathrm{MLS}_{\mathrm{B}}\right)$ is increasingly used because of their excellent oral absorption and decent tissue penetration as well as their ability to accumulates in abscesses. This has made it the best choice in both veterinary and human medicine [19] [20] [21]. Clindamycin is an antimicrobial agent which belong to the Macrolide-Lincosamide-Streptogramin $\mathrm{B}\left(\mathrm{MLS}_{\mathrm{B}}\right)$ family. The wide spread use of the $\mathrm{MLS}_{\mathrm{B}}$ family of antimicrobials has led to the emergence of resistance. Our study showed prevalence of induced Macrolide-Lincosamide-Streptogramin B ( $\mathrm{iMLS}_{\mathrm{B}}$ ) among MRSA isolated from both Human and swine $42 \%$, which is in agreement with other reports elsewhere [21] [22] [23]. Higher rates of $\mathrm{iMLS}_{\mathrm{B}}$ have been reported in other studies conducted in Uganda [24] and Kenya [25]. Molecular studies have indicated that some SCCmec elements on MRSA may carry transposon Tn554 which contains the gene ermA mediating MLS resistance [23]. This could be a probable reason for higher rate of resistance reported in our study. MRSA isolates from human had higher iMLSB compared to those isolated from swine. This suggests an increasing usage of this class of antibiotics resulting selective pressure and consequently 
multidrug resistance [26]. Probable widespread empirical use of erythromycin and clindamycin today and increasing consumption are the drivers of selective pressure [27]. We suggest reduction in macrolide usage to reverse such resistance pattern as it has been described elsewhere [28].

Clindamycin resistance can develop in the Staphylococcal bacteria with the inducible phenotype and spontaneous constitutive resistant mutants can also be selected from iMLSB isolates. This may happen both in vitro and in vivo during the CL treatment and this phenomenon is faster in the MRSA strains [6] [7]. We report a high prevalence of cMLSB [48\%] among all the MRSA isolates with high percentage registered swine isolates [47\%]. These findings are in agreement with several other studies by Almasri et al. (2016) and Ganesh et al. (2016) who reported that constitutive phenotype is predominant higher than inducible phenotype in MRSA isolates [29] [30]. However, different results have been reported in India, where Kumari et al. (2016) reported $\mathrm{cMLS}_{\mathrm{B}}(35.2 \%)$ and $\mathrm{iMLS}_{\mathrm{B}}(15.9 \%)$ showing higher constitutive resistance among MRSA [31]. In similar note, Das et al. (2016) showed that cMLSB was $36.8 \%$ compare to iMLSB of $31.8 \%$ in MRSA isolates [19]. Also, Mohammad, 2012 reported 32.5\% of MRSA were cMLSB phenotypes and $10 \%$ were iMLSB phenotype. These variations could probably be due to differences in the circulating clones or due to the variations in infection prevention practices and trends of antibiotics prescriptions in the community and veterinary practice [32].

The current study also reveals $6 \%$ of MS phenotype (E-R, Cl-S) among MRSA isolates. In this case, clindamycin can be used as treatment option only for less number of MRSA which are erythromycin resistant. While treating Erythromycin MRSA infection with Clindamycin antibiotic, there is always minimum chance of clinical efficacy compared to vancomycin antibiotic therapy [33]. We therefore emphasize routine use of D-test in diagnostic laboratories to avoid clinical failure while using clindamycin as an alternative to anti-MRSA antibiotics like vancomycin and linezolid [34] [35].

\section{Conclusion}

The prevalence of inducible and constitutive clindamycin resistance among MRSA Isolates from both humans and swine is high. The D test is a simple and affordable technique that can be used in low resourced settings to define precisely MLSB, both inducible and constitutive resistance patterns in addition to MSB in Staphylococcus aureus in the routine clinical laboratories. This can be an important strategy for good antibiotic stewardship in under resourced settings.

\section{Conflicts of Interest}

The authors declare no conflicts of interest regarding the publication of this paper.

\section{References}

[1] Asiimwe, B.B., Baldan, R., Trovato, A. and Cirillo, D.M. (2017) Molecular Epidemi- 
ology of Panton-Valentine Leukocidin-Positive Resistant Staphylococcus aureus Isolates in Pastoral Communities of Rural South Western Uganda. BMC Infectious Diseases, 17, 24. https://doi.org/10.1186/s12879-016-2124-8

[2] Mahon, C.R., Donald, C. and Lehman, G.M. (2015) A Textbook of Diagnostic Microbiology. 5th Edition, Elsevier, Amsterdam, 254-273.

[3] Steward, C.D., Raney, P.M., Morrell, A.K., Williams, P.P., Mcdougal, L.K. and Jevitt, L. (2005) Testing for Induction of Clindamycin Resistance in Erythromycin-Resistant Isolates of Staphylococcus aureus. Journal of Clinical Microbiology, 43, 17161721. https://doi.org/10.1128/JCM.43.4.1716-1721.2005

[4] Gaynor, M. and Mankin, A.S. (2003) Macrolide Antibiotics: Binding Site, Mechanism of Action, Resistance. Current Topics in Medicinal Chemistry, 3, 949-961. https://doi.org/10.2174/1568026033452159

[5] Morar, M., Bhullar, K., Hughes, D.W., Junop, M. and Wright, G.D. (2009) Structure and Mechanism of the Lincosamide Antibiotic Adenylyltransferase LinB. Structure, 17, 1649-1659. https://doi.org/10.1016/j.str.2009.10.013

[6] Bouazza, N., Pestre, V., Jullien, V. and Curis, E. (2012) Population Pharmacokinetics of Clindamycin Orally and Intravenously Administered in Patients with Osteomyelitis. British Journal of Clinical Pharmacology, 74, 971-977. https://doi.org/10.1111/j.1365-2125.2012.04292.x

[7] Reddy, P.S. and Suresh, R. (2012) Phenotypic Detection of Inducible Clindamycin Resistance among the Clinical Isolates of Staphylococcus aureus by Using the Lower Limit of Inter Disk Space. Journal of Microbiology and Biotechnology Research, 2, 258-264.

[8] Teeraputon, S., Santanirand, P., Wongchai, T., Songjang, W., Lapsomthob, N. and Jaikrasun, D. (2017) Prevalence of Methicillin Resistance and Macrolide-Lincosamide-Streptogramin B Resistance in Staphylococcus Haemolyticus among Clinical Strains at a Tertiary-Care Hospital in Thailand. New Microbes and New Infections, 19, 28-33. https://doi.org/10.1016/j.nmni.2017.05.007

[9] Prabhu, K., Rao, S. and Rao, V. (2011) Inducible Clindamycin Resistance in Staphylococcus aureus Isolated from Clinical Samples. Journal of Laboratory Physicians, 3, 25-27. https://doi.org/10.4103/0974-2727.78558

[10] Murphy, E. (1985) Nucleotide Sequence of ermA, a Macrolide-Lincosamide-Streptogramin B Determinant in S. aureus. Journal of Bacteriology, 162, 633-640.

[11] Reygaert, W.C. (2013) Antimicrobial Resistance Mechanisms of Staphylococcus aureus. In: Nendez-Vilas, A., Ed., Microbial Pathogens and Strategies for Combating Them: Science, Technology and Education, Formatex Research Center, Badajoz, Spain, 297-305.

[12] Piątkowska, E., Piątkowski, J. and Przondo-Mordarska, A. (2012) The Strongest Resistance of Staphylococcus aureus to Erythromycin Is Caused by Decreasing Uptake of the Antibiotic into the Cells. Cellular and Molecular Biology Letters, 17, 633-645. https://doi.org/10.2478/s11658-012-0034-3

[13] Levin, T.P., Suh, B., Axelrod, P., Truant, A.L. and Fekete, T. (2005) Potential Clindamycin Resistance in Clindamycin-Susceptible, Erythromycin-Resistant Staphylococcus aureus. Report of a Clinical Failure. Antimicrobial Agents and Chemotherapy, 49, 1222-1224. https://doi.org/10.1128/AAC.49.3.1222-1224.2005

[14] Mokta, K.K., Verma, S., Chauhan, D. and Ganju, S.A. (2015) Inducible Clindamycin Resistance among Clinical Isolates of Staphylococcus aureus from Sub Himalayan Region of India. Journal of Clinical and Diagnostic Research, 9, 20-23. https://doi.org/10.7860/JCDR/2015/13846.6382 
[15] Waites, K.E.N., Johnson, C., Gray, B., Edwards, K., Crain, M. and Benjamin, W. (2000) Use of Clindamycin Disks to Detect Macrolide Resistance Mediated by ermB and mefE in Streptococcus pneumoniae Isolates from Adults and Children. Journal of Clinical Microbiology, 38, 1731-1734.

[16] Datta, P., Gulati, N., Singla, N., Vasdeva, H.R. and Bala, K. (2017) Evaluation of Various Methods for the Detection of Meticillin-Resistant Staphylococcus aureus Strains and Susceptibility Patterns. Journal of Medical Microbiology, 60, 1613-1616. https://doi.org/10.1099/jmm.0.032219-0

[17] Queipo-Ortuño, M.I., De Dios Colmenero, J., Macias, M., Bravo, M.J. and Morata, P. (2008) Preparation of Bacterial DNA Template by Boiling and Effect of Immunoglobulin $\mathrm{g}$ as an Inhibitor in Real-Time PCR for Serum Samples from Patients with Brucellosis. Clinical and Vaccine Immunology, 15, 293-296.

https://doi.org/10.1128/CVI.00270-07

[18] Rodríguez-Noriega, E., Seas, C., Guzmán-Blanco, M., Mejía, C., Alvarez, C., Bavestrello, L., et al. (2010) Evolution of Methicillin-Resistant Staphylococcus aureus Clones in Latin America. International Journal of Infectious Diseases, 14, 560-566.

https://doi.org/10.1016/j.ijid.2009.08.018

[19] Das, P.P. and Choudhury, G.S.L. (2016) Inducible Clindamycin Resistance in Clinical Isolates of Staphylococcus Aureus. International Journal of Medical Research Professionals, 2, 65-69.

[20] Mendes, R.E., Castanheira, M., Farrell, D.J., Flamm, R.K., Sader, H.S. and Jones, R.N. (2017) Prevalence of Macrolide-Lincosamide Resistance and Multidrug Resistance Phenotypes in Streptococcal Isolates Causing Infections in European Hospitals. Journal of Global Antimicrobial Resistance, 8, 28-32. https://doi.org/10.1016/j.jgar.2016.08.013

[21] Marosevic, D., Kaevska, M. and Jaglic, Z. (2017) Resistance to the Tetracyclines and Macrolide-Lincosamide-Streptogramin Group of Antibiotics and Its Genetic Linkage: A Review. Annals of Agricultural and Environmental Medicine, 24, 338-344. https://doi.org/10.26444/aaem/74718

[22] Mshana, S.E., Kamugisha, E., Mirambo, M., Chalya, P., Rambau, P. and Mahalu, W. (2009) Prevalence of Clindamycin Inducible Resistance among Methicillin-Resistant Staphylococcus aureus at Bugando Medical Centre, Mwanza, Tanzania. Tanzania Journal of Health Research, 11, 59-64. https://doi.org/10.4314/thrb.v11i2.45197

[23] Ammar, A., Attia, A., Abd El-Hamid, M., El-Shorbagy, I. and Abd El-Kader, S. (2016) Genetic Basis of Resistance Waves among Methicillin Resistant Staphylococcus aureus Isolated Recovered from Milk and Meat Products in Egypt. Cellular and Molecular Biology, 62, 7-15.

[24] Mwambi, B., Iramiot, J., Bwanga, F., Nakaye, M., Itabangi, H. and Bazira, J. (2014) Clindamycin Resistance among Staphylococcus aureus Isolated at Mbarara Regional Referral Hospital, in South Western Uganda. British Microbiology Research Journal, 4, 1335-1344. https://doi.org/10.9734/BMRJ/2014/10572

[25] Maina, E.K., Kiiyukia, C., Wamae, C.N., Waiyaki, P.G. and Kariuki, S. (2013) Characterization of Methicillin-Resistant Staphylococcus aureus from Skin and Soft Tissue Infections in Patients in Nairobi, Kenya. International Journal of Infectious Diseases, 17, 115-119. https://doi.org/10.1016/j.ijid.2012.09.006

[26] Feltrin, F., Alba, P., Kraushaar, B., Ianzano, A., Argudín, A. and Matteo, D. (2016) Staphylococcus aureus Clonal Complex 97 Lineage Spreading in Dairy Cattle and Pigs in Italy. Applied and Environmental Microbiology, 82, 816-821. 
https://doi.org/10.1128/AEM.02854-15

[27] Dubey, D., Rath, S., Sahu, M.C., Rout, S., Debata, N.K. and Padhy, R.N. (2013) A Report on Infection Dynamics of Inducible Clindamycin Resistance of Staphylococcus aureus Isolated from a Teaching Hospital in India. Asian Pacific Journal of Tropical Biomedicine, 3, 148-153. https://doi.org/10.1016/S2221-1691(13)60040-4

[28] Seppälä, H., Klaukka, T., Vuopio-Varkila, J., Muotiala, A., Helenius, H., Lager, K., et al. (1997) The Effect of Changes in the Consumption of Macrolide Antibiotics on Erythromycin Resistance in Group A Streptococci in Finland. The New England Journal of Medicine, 337, 441-446. https://doi.org/10.1056/NEJM199708143370701

[29] Almasri, M., Abu Hasan, N. and Sabbah, N. (2016) Macrolide and Lincosamide Resistance in Staphylococcal Clinical Isolates in Nablus, Palestine. Turkish Journal of Medical Sciences, 46, 1064-1070. https://doi.org/10.3906/sag-1503-121

[30] Singh, G.K., Chaudhari, B.K. and Parajuli, K.P. (2016) Phenotypic Study of Macrolide-Lincosamide-Streptogramin B Resistance in Staphylococcus aureus and Their Relationship with Methicillin-Resistant Staphylococcus aureus (MRSA) at Tertiary Care in Eastern Nepal. Journal of Nobel Medical College, 5, 1-5. https://doi.org/10.3126/jonmc.v5i1.15745

[31] Kumari, J., Shenoy, S.M., Baliga, S., Chakrapani, M. and Bhat, G.K. (2016) Healthcare-Associated Methicillin-Resistant Staphylococcus aureus. Clinical Characteristics and Antibiotic Resistance Profile with Emphasis on Macrolide-LincosamideStreptogramin B Resistance. Sultan Qaboos University Medical Journal, 16, 175-181. https://doi.org/10.18295/squmj.2016.16.02.007

[32] Ferreira, S.B., Martins, J.R.T.L., Ferreira, P.B. and Lima, Z.N. (2015) Induced and Constitutive Clindamycin Resistance in Staphylococcus spp. Strains Isolated from a Neonatal Intensive Care Unit. International Journal of Tropical Disease \& Health, 9, 1-8. https://doi.org/10.9734/IJTDH/2015/18522

[33] Vandana, K., Singh, J., Chiranjay, M. and Bairy, I. (2009) Inducible Clindamycin Resistance in Staphylococcus aureus. Reason for Treatment Failure. Journal of Global Infectious Diseases, 1, 76.

[34] Patel, M., Waites, K.B., Moser, S.A., Cloud, G.A. and Hoesley, C.J. (2006) Prevalence of Inducible Clindamycin Resistance among Community- and Hospital-Associated Staphylococcus aureus Isolates. Journal of Clinical Microbiology, 44, 24812484. https://doi.org/10.1128/JCM.02582-05

[35] Vaibhav, S. and Tathe, S. (2015) Phenotypic Detection and Incidence of Inducible Clindamycin Resistance among Staphylococcus aureus from Tertiary Care Hospital. International Journal of Advances in Medicine, 2, 264-268.

https://doi.org/10.18203/2349-3933.ijam20150557 